\title{
A microphotometer for measuring luminance distributions on a CRT
}

\author{
VEIJO VIRSU and P. K. LEHTIÖ \\ University of Helsinki, SFO01 70 Helsinki 17. Finland
}

\begin{abstract}
A relatively inexpensive microphotometer is described. The output voltage of the microphotometer is a linear function of luminance, and the sensitivity of the photometer is sufficient for the measurement spot size of $.2 \mathrm{~mm}$ without special averaging devices. The rise and decay time of the photometer response is less than 100 microsec, and the spectral sensitivity of the photometer can be corrected to approximate that of the human photopic eye.
\end{abstract}

Cathode-ray tubes (CRTs) are convenient devices for producing well-controlled visual stimuli if one wants to vary the spatial or temporal features of the stimulus. A large range of different stimulus patterns, such as gratings, circles, angles, point intervals, line segments, rectangles, etc., can be drawn in $x-y-z$ mode with function generators. A desired feature, such as size, angle, luminance, contrast, duration, velocity of movement, flicker rate, etc., can be controlled by an observer or experimenter through simple potentiometer circuits, and the adjustments can be monitored accurately from digital voltmeters. Complex experiments can be performed with the system without computers, but it is easy to interface a computer to the fully electronic controls of CRT, and this increases the versatility of the system even further.

The most inconvenient feature of a CRT stimulation system is that the stimuli are generated on a surface that has an inaccessible position behind a thick glass; hence, the calibration of stimuli is difficult. When accurate calibrations are needed, the only solution is to use a microphotometer that can be focused onto the phosphor. Commercial microphotometers are available, but they are very expensive and few of them can be easily adapted for calibrations required in psychophysical and physiological experiments. We have designed and built a simple microphotometer that is relatively inexpensive and still adequate for various calibration tasks. Some of our experiences are reported below.

\section{DESIGN}

Figure 1 shows a schematic illustration of the photometer, which consists of three main parts: microscope (M), extension tube (T), and housing for the light-sensitive element $(\mathrm{H})$. An image presented on phosphor (p) enters the microscope through a circular, knife-edged aperture (a) and objective (o). The image reaches its full magnification after it leaves the

This research was supported by SITRA, Contract No. 6025 and by the Finnish Academy. We thank Seppo Lindberg and Pauli Visuri for building the microphotometer and Marie-Louise Tiderman for help in preparation of the manuscript.

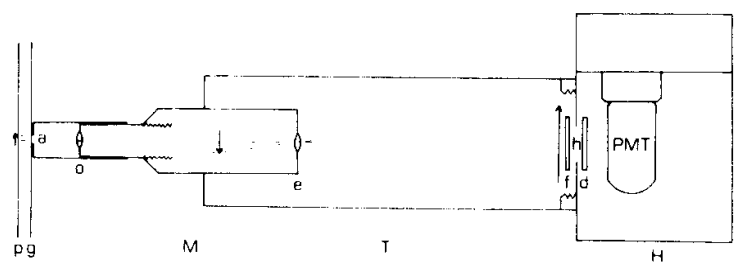

Figure 1. Schematic illustration of the microphotometer. See text for details.

microscope ocular (e) and is focused for a sharp projection at a circular hole (h) through which light enters the housing containing a photomultiplier tube (PMT). A diffuser (d) scatters the light so that the directional properties of the PMT do not affect the measurements. A filter (f) can be placed in front of the diffuser for adjusting the spectral sensitivity of the photometer. The photometer dynode circuit and an amplifier for converting current responses to voltages are fitted into a diecast aluminum box housing also the PMT.

An inexpensive microscope tube with about $50 \mathrm{x}$ magnification and $25 \mathrm{~mm}$ focal length of the objective is adequate for most purposes. A movable socket carrying the aperture is fitted about the objective holder so that when the focus of the microscope is correct, the aperature (a) is placed against the front glass $(\mathrm{g})$ of the CRT to be measured. A standard set of camera extension rings and extension bellows can be used for building the extension tube; it is easy to fix the microscope and the case containing the PMT to the extension rings, which in turn are screwed to the extension bellows. The focus of the microscope on the phosphor can be adjusted visually when one of the ring joints is opened. If the accommodation of the eye is relaxed during focusing so that the eye is focused to infinity, the resulting focus of the microscope is satisfactory for most purposes. If more accurate focusing is desired, a lens can be added next to the ocular after visual focusing. The focal length of the lens should be about the same as the distance between the ocular and the diffuser.

In our photometer, aperture (a) was $1 \mathrm{~mm}$ in diam. The function of the aperture is to decrease the amount 
of scattered light resulting from internal reflections of side light in the objective. The distance between the ocular and the diffuser was $17 \mathrm{~cm}$. Opening $h$ in front of the diffuser had two alternative sizes, 5 and $10 \mathrm{~mm}$ in diam.

There are several inexpensive PMTs that are adequate for serving as the light-sensitive element. We chose the RCA 1P28A, which has nine stages. Its dynode circuit was built following the manufacturer's suggestions (RCA, PIT-700B).

The preamplifier circuit is shown in Figure 2. It was built by using an operational amplifier as a current-to-voltage transducer (cf. Keene, Black, \& Hayon, 1969.) We used an AD523J (Analog Devices) low bias current amplifier with FET input stage. The power supply for the preamplifier was built into a separate case. The supply leads were shunted to ground with 1,000 microF and 100 microF capacitors near the case of the amplifier. The amplifier converts the linear anode-current response of the PMT to light flux into a linear voltage response, the slope of which depends on feedback resistance ( $10 \mathrm{kohm}$ in our photometer). Brandenburg Model 476R serves as our high-voltage supply for the PMT.

The voltage response to light can be recorded in various ways. If low luminance levels or small differences are recorded, accurate averaging may become necessary and a computer is most suitable for this task. In all problems we have encountered so far, however, an oscilloscope has been adequate for recording, and whenever a need for averaging has arisen, an oscilloscope with a storage display has given the means for accurate-enough visual averaging with the aid of a movable reference line. A low-pass filter $(-3 \mathrm{~dB} / 3 \mathrm{kHz})$ facilitates oscilloscope reading but is not necessary.

\section{PROPERTIES OF THE PHOTOMETER}

It is necessary to keep in mind when measuring the luminance of an image on a CRT that the visible image is an illusion. There is only a fast-moving luminous spot whose trace decays very quickly. The permanent appearance of image results from the persistence of the visual effect caused by the moving spot. As a consequence, the luminance of the moving spot alone does not specify the luminance of the image, but it depends on several factors such as velocity of the spot, area covered by the image, decay characteristics of the phosphor, frame frequency, and so on. The luminance of an image on a CRT has to be measured so that the parameters of temporal and spatial integration are the same in the photometer and in the eye, in addition to similar spectral sensitivity, because the visual effects of light are measured in photometry.

It is not possible to design a general-purpose microphotometer that would fulfill the requirements above because the concept of microphotometer excludes a match in spatial integration properties. In the other

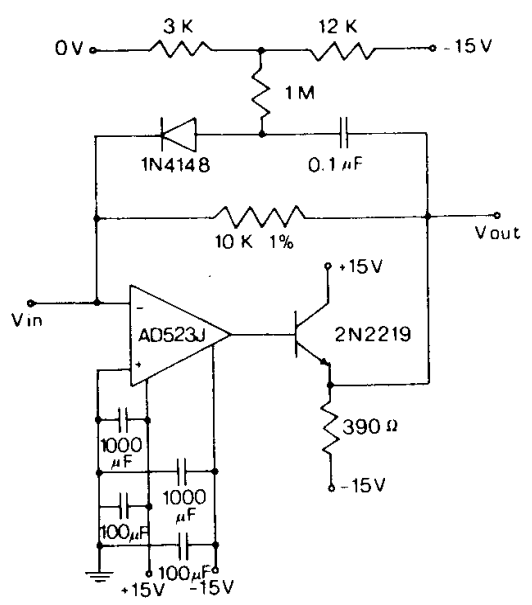

Figure 2. Photomultiplier preamplifier.

hand, temporal resolution of the photometer should be abandoned for getting a match in temporal integration. Neither spatial nor temporal integration properties of the eye are constants, for they vary as a function of adaptation level and depend also on several other factors. Therefore, a proper goal for the design of a microphotometer is that it is suitable for relative measurements.

If absolute measurements of CRT luminances are required, the photometer has to be calibrated individually for every new experimental arrangement. Our procedure has been such that we have calibrated a Salford Instruments SEI "subjective" photometer against a Standard Illuminant A source and diffuser. An image suitable for accurate measurements with the SEI photometer has been generated on the CRT, using the intended frame frequency and spot velocity. Its luminance has given us a calibration reference for interpreting the voltage output of the microphotometer in terms of equivalent luminance. This procedure is easy to apply when one works with stimuli generated on a raster basis because the raster forms a calibration reference. If one works with line figures produced on a CRT so that the beam velocity is not a constant and wants to make absolute measurements, temporal averaging of the photometer output is required. This can be performed by a computer or through a simple averaging circuit added to the photometer. It is important to note, however, that temporal averaging destroys the temporal resolution of the photometer, which is useful in many applications and requires a decision regarding the time constant of averaging. Therefore, any temporal averaging device should be kept as a switch-selectable option.

The photometer can be given a permanent calibration with respect to any constant source of light. Figure $3 \mathrm{~A}$ shows the voltage output of our photometer as a function of the luminance of a white surface. A diffuse Plexiglas screen was transilluminated with a projector having a tungsten-iodine lamp; neutral-density filters 

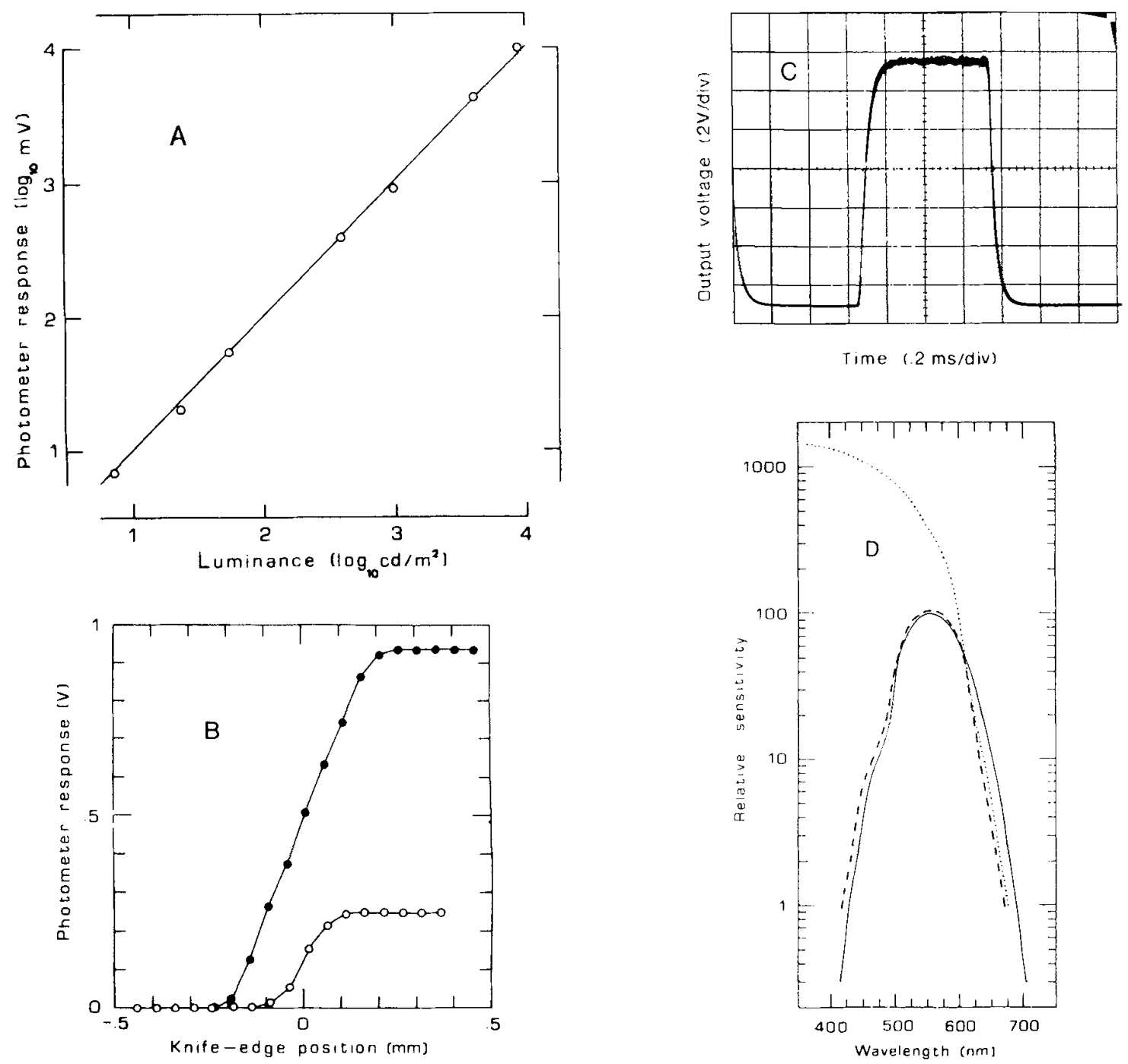

Figure 3. Properties of the photometer. (A) Voltage output as a function of field luminance. (B) Spatial response at two sizes of the diffuser window. Filled-in circles, 10-mm window; open circles, 5-mm window. (C) Temporal response shown by an oscilloscope trace. (D) Relative spectral response calculated from manufacturer's data. Dotted line, RCA 1P28A; broken line, Kodak Wrat ten No. 106 filter added; continuous line, the photopic luminosity function for the CIE standard observer.

were used for attenuating the light of the projector. The supply voltage to PMT was $500 \mathrm{~V}$. The photometer output was a linear function of luminance from 8 to $10,000 \mathrm{~cd} / \mathrm{m}^{2}$ as measured with the SEI photometer and calculated also from the density values of the filters so that $1 \mathrm{mV}$ corresponds to $1 \mathrm{~cd} / \mathrm{m}^{2}$. The diffuser window (h) of the photometer was $10 \mathrm{~mm}$. Down to the value of $8 \mathrm{~cd} / \mathrm{m}^{2}$, visual averaging was sufficient to retain a reading accuracy better than $\pm 10 \%$. Lower levels of luminance could have been recorded with a higher supply voltage, but then the whole range of measurable luminances would have been lower and a signal averager would have become necessary for recording the lower values of luminance.

Figure 3B shows the spatial response of the photometer. Using a micro-manipulator, the smoked edge of a razor blade was moved in front of the photometer receptive area when the occluding edge was in sharp focus. The background light was provided by a $50-\mathrm{Hz}$ raster generated on an HP1300A display with white P4 phosphor. The raster was homogenous and consisted of 1,000 lines per $25 \mathrm{~cm}$ so that no dark space remained between the lines. The mean luminance of the raster was $14 \mathrm{~cd} / \mathrm{m}^{2}$, assessed with the SEI photometer. As is seen from the fillea-in circles, the photometer response rose from 0 to a maximum value of $.95 \mathrm{~V}$ when the occluding edge moved about $4 \mathrm{~mm}$ and the diffuser window was $10 \mathrm{~mm}$ in diam. Thus, the size of the measuring spot was about $.4 \mathrm{~mm}$. The spot was then slightly larger than the width of an individual raster line and, therefore, one can conclude that the luminance of the spot created by the electron beam was of the order of $1,000 \mathrm{~cd} / \mathrm{m}^{2}$ because $1 \mathrm{~V}$ of photometer response to white light corresponds to $1,000 \mathrm{~cd} / \mathrm{m}^{2}$ (see Figure 3A). 


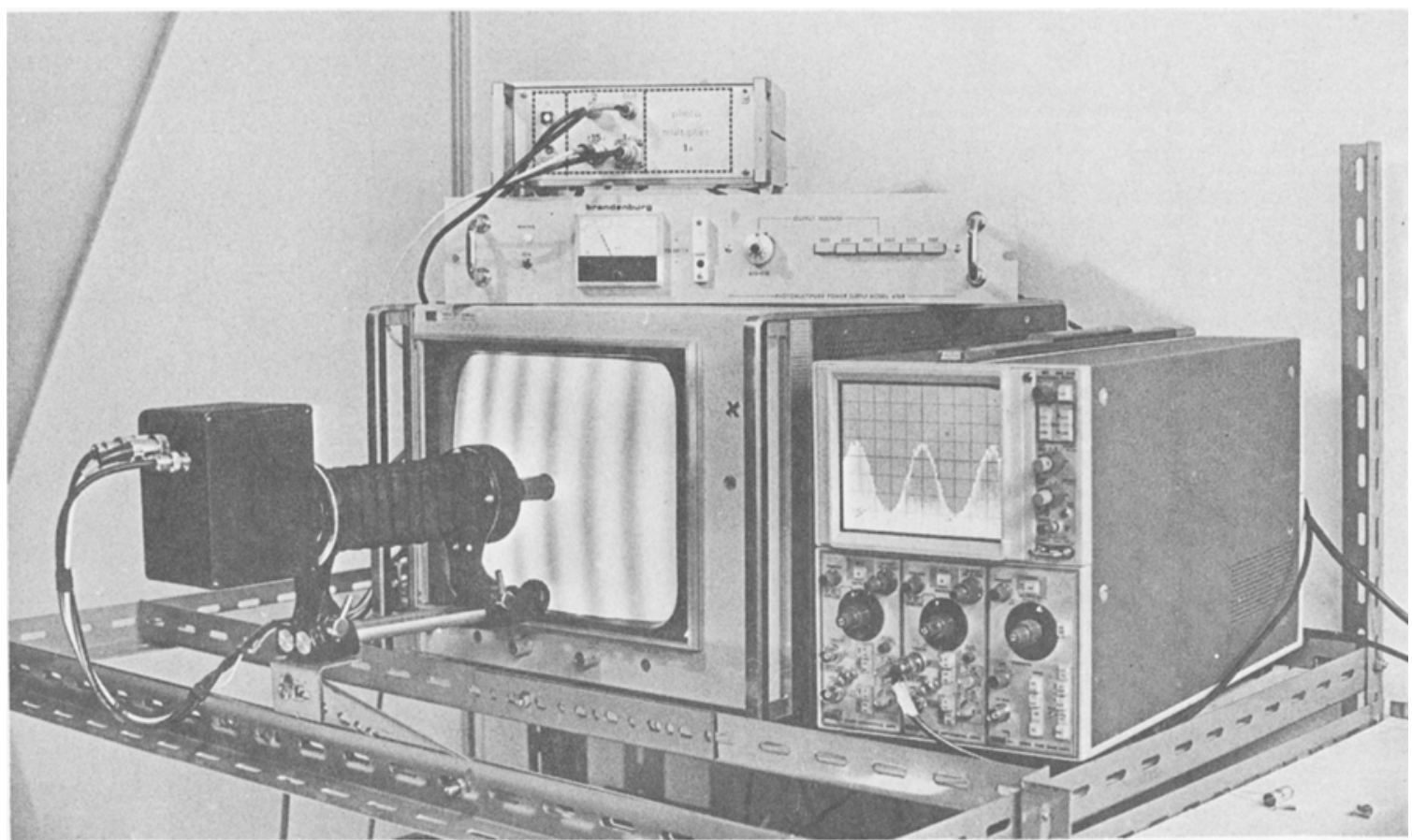

Figure 4. Monitoring the waveform, contrast, and velocity of movement of a grating with the microphotometer (on the left). The voltage supplies for the photometer are seen above the display on which a drifting grating is drawn. One trace of the photometer output has been stored on the oscilloscope screen on the right.

The open circles show the effect of inserting a $5-\mathrm{mm}$ opening in front of the diffuser. The measurement spot was now about $.2 \mathrm{~mm}$ in diam, and the sensitivity of the photometer decreased about the expected $12 \mathrm{~dB}(75 \%)$ from the value obtained with the $10-\mathrm{mm}$ opening. The orientation of the raster lines was the same as the direction of the razor-blade movement. Therefore, the measured spatial response is accurate and representative for CRT applications. As the net magnification of the microscope depends on the distance between the objective and the phosphor, the only reliable method for determining the spatial response is to measure it empirically.

Figure 3C shows the temporal response of the photometer. A diffuse Plexiglas screen was transilluminated by a $6-\mathrm{V}$ tungsten lamp, driven by a voltage of $8 \mathrm{~V} \mathrm{dc}$, and a chopper disk having two $15-\mathrm{mm}$ openings was rotated in front of the photometer entrance pupil. The diffuser window was $10 \mathrm{~mm}$. The oscillogram shows about 20 superimposed traces. The rise and decay time of the photometer are both less than 100 microsec. No temporal filter was used; note the high-frequency noise at the top of the oscillogram. The filtering action of $3 \mathrm{~m}$ of $75-\mathrm{ohm}$ coaxial cable is included in the response, however.

Figure 3D shows the approximate spectral sensitivity of the RCA 1P28A photomultiplier as specified by the manufacturer. The broken line shows the calculated spectral sensitivity resulting if Kodak Wratten Gelatin
Filter No. 106 is inserted in the light path. The continuous line shows the CIE photopic spectral sensitivity of the human eye. For many purposes, correction by the No. 106 filter would be satisfactory, although it is evident that the match would not be accurate. With $\mathrm{P} 4$ phosphor, No. 106 filter decreases the sensitivity of the photometer by about $83 \%$. The measurements shown in Figures $3 \mathrm{~A}-\mathrm{C}$ were made without spectral correction.

\section{APPLICATIONS OF THE PHOTOMETER}

It is clear that different applications require different characteristics from the photometer. Any of the characteristics above can be improved when required by a particular application, but then, of course, some other requirements must be given up. Sensitivity and $\mathrm{S} / \mathrm{N}$ ratio decrease if a smaller measurement spot or better spectral response are required. If spectral response is of importance, it might be better to select some other type of PMT whose spectral response should be individually corrected taking into account also the spectral effects of the microscope.

The overall characteristics of the photometer can be improved if the price of the photometer is allowed to increase. The cost of parts of our photometer was about $\$ 250.00$ plus the two power supplies, about $\$ 700.00$ altogether. The prices of commercial microphotometers that could fulfill similar purposes start at about 
$\$ 5,000.00$. The absolute sensitivity of the photometer can be increased very much if a higher supply voltage is applied and the photometer output is averaged by a computer or by a signal averager. If one wants to measure the luminance of line figures on a CRT in absolute units, an inexpensive temporal averaging circuit can be added to the photometer itself. So far, we have not encountered a situation requiring these additional devices, however.

Without improvements, our photometer is fully adequate for measuring the transfer function of a display system and for calibrating all the variables concerned when working with grating stimuli. Figure 4 shows the basic arrangement when gratings are calibrated. With a $5-\mathrm{mm}$ diffuser window, the $2-\mathrm{mm}$ measuring area is small enough for calibrating the contrast values of the finest gratings that can be produced on an ordinary CRT. A grating is moved in Figure 4, and its luminance profile is reproduced on the oscillosiope screen. Alternatively, the photometer can be moved if it is fixed to a micromanipulator. The luminance minima and maxima can be read directly from? the oscilloscope screen. If a reference line is added on the screen for visual averaging, contrast can be read down to .01. As the photometer output is proportional to luminance, a need to convert the voltage output into luminance units occurs very seldom. If luminance values are desired, the voltage-to-luminance conversion can be made by measuring output level at /ero contrast.
An initial warm-up period of $2 \mathrm{~h}$ is enough to stabilize drift due to temperature, and the stability of the photometer output has proved excellent when measuring CRT images. So far, we have measured the following variables with the photometer: CRT luminance/voltage transfer, spatial and temporal modulation transfer of the display system, luminance waveforms of gratings in time and space, mean luminance and contrast for gratings drawn in different orientations, velocity of grating movements, and noise of the display systems. These measurements have been worthwhile, for the transfer functions of the display systems have turned out to contain various kinds of non-linearities such that one may crr considerably in any linear interpolation.

If the photometer is fixed to a micromanipulator, it is possible to measure the spatial distribution of luminance on any back-illuminated surface with the photometer. The good temporal resolution of the photometer makes it well-suited also for accurate determination of temporal events in various stimulus-production systems.

\section{REFERENCES}

Keene, J. P., Black, E. D., \& Hayon, E. A photomultiplier and amplifier circuit for kinetic spectrophotometry. The Review of Scientific Instruments. 1969, 40, 1199-1201.

RCA. Photomultiplier tubes. PIT-700B 12/71. RCA, Electronic Componen is, Harrison, N.J., 1971.

(Received for publication October 20, 1974; revision received December 4,1974 .) 